$\xi=-1$

\title{
Investigation on the Effect of Centrifugation Speed on the Shape Separation of Gold Nanorods
}

\author{
Caessandra Addine Minun ${ }^{1,4}$, Nur Zehan An’Nisa ${ }^{1,2}$, Marlia Morsin ${ }^{1,2}$, Nur Liyana Razali ${ }^{1,2}$, Suratun Nafisah ${ }^{1,2}$ \\ Rahmat Sanudin $^{1,2}$, Muhamad Mat Salleh ${ }^{3}$ \\ 1Microelectronics \& Nanotechnology - Shamsuddin Research Centre (MiNT-SRC), Institute of Integrated Engineering (I2E), \\ Universiti Tun Hussein Onn Malaysia, 86400 Parit Raja, Batu Pahat Johor, Malaysia. \\ 2Department of Electronics, Faculty of Electrical and Electronics Engineering, Universiti Tun Hussein Onn Malaysia, \\ 86400 Parit Raja, Batu Pahat Johor, Malaysia \\ 3Institute of Microengineering \& Nanoelectronics (IMEN), Universiti Kebangsaan Malaysia, 43600 Bangi, Selangor, Malaysia. \\ 4Intel Technology (M) Sdn. Bhd, Bayan Lepas Free Trade Zone, 11900 Penang, Malaysia. \\ *Corresponding author E-mail: marlia@uthm.edu.my
}

\begin{abstract}
High-quality gold nanorods (AuNRs) with a uniform aspect ratio and sizes are crucial for applications in biomedical or sensor industry. AuNRs is implementing Localized Surface Plasmon Resonance (LSPR) as sensing method which is highly dependent on the size, separation of nanoparticles, aspect ratio and the shape of the nanoparticles. Thus, in this process, the shape, surface density and aspect ratio of AuNRs were investigated to be used further as LSPR sensing material. The AuNRs was prepared using Seed Mediated Growth Method (SMGM) and the growth solution of the AuNRs was centrifuged with the speed ranging from $2500 \mathrm{rpm}$ to 5000 rpm to separate the particles shaper prior to deposition as thin film. The XRD result shows the peak at $2 \theta=38^{\circ}$ which shows the (111) crystal orientation of the AuNRs. There are also two distinct peaks formed at the absorption spectra graph obtained from the UV-Vis result which associated with transverse plasmon peak (t-LSPR) and longitudinal plasmon peak (1-LSPR). The t-LSPR peak is significantly smaller than the 1-LSPR and located in the wavelength ranges from $500 \mathrm{~nm}$ to $560 \mathrm{~nm}$ whereas the l-LSPR of the AuNRs shows a stronger and a higher peak which is located in the wavelength ranges from $700 \mathrm{~nm}$ to $780 \mathrm{~nm}$. The morphological analysis using FESEM shows that the higher centrifugation speed separates the AuNRs with higher aspect ratio and higher surface density.
\end{abstract}

Keywords: Centrifugation; Gold Nanorod, Separation Speed; Localized Surface Plasmon Resonance; Plasmonic Sensor.

\section{Introduction}

Gold nanoparticles (AuNPs) is a noble metal that exhibits extraordinary properties that portray unique optical properties which originate from the excitation of their localized surface plasmon resonance (LSPR) modes [1]-[3]. Due to their interactions between the conduction bands and lights, the AuNPs are considered as promising nanoparticles for the application industry. Synthesizing and separating metal AuNPs in terms of its shape and sizes are the most challenging and important tasks. The size can influence the optical properties of the metal nanoparticles. In both spheroidal or rod-shaped gold nanoparticles, the similarities of these two shapes to the spherical particles is the appearance of their surface plasmon at a lower energy [4]. Noble materials such as metal nanoparticles display some unique optical properties due to the excitation of localized surface plasmon resonance (LPSR) modes. They also flaunt a strong UV-visible absorption band which is not shown in the spectrum of the bulk metal [5]. AuNPs possess various sizes varying from $1 \mathrm{um}$ to $8 \mathrm{um}$ and also can be synthesized into different shapes such as spherical, rod and many more. Gold nanospheres (AuNSs) are commonly used in many fields [6] but it has some disadvantages such as their surface plasmon properties exhibit only one plasmon band [7] in the near infrared area and thus restrict their application in the sensing field compared to gold nanorods (AuNRs)
Gold nanorods (AuNRs) are nanocrystals with non-spherical and rod-like shape and obtain so much attention because of their important application in optical devices [7], biochemical sensors [8] and nanomedicine [3]. Besides, in biomedical imaging, it could penetrate deep into tissues and cells due to their tunable optical response near-infrared wavelengths [9]. AuNRs are popular for their unique optical properties which can be approximated by the Mie-Gans theory. They also have two SPR bands [3] which will occur depending on the polarization of the incident light. These two bands are called the transverse (short axis) and longitudinal (long axis) band. Transverse band happens when the excitation occurs along the shorter axis and it induces absorption in the visible region whereas the longitudinal band induces a much stronger absorption band along the longer axis and much more sensitive to the aspect ratio of the AuNRs compared to the transverse band [7], [10].

Multiple of growth methods have been investigated throughout the past two decades to develop the AuNRs with the goals to improve the yield, aspect ratio, shape and homogeneity. AuNRs also can be assembled into some number of superstructures which results in a greater plasmonic properties. For synthesising AuNRs, some of the methods used such as Turkevish method [11], Burst Schiffrin method [12], [13], electrochemical method and also seed mediated growth method (SMGM) [14]. The SMGM is used in this research because it synthesizes high quality of AuNRs and the LSPR of AuNRs can be tunable by changing the aspect ratio of the AuNRs. 
SMGM also produces AuNRs with higher surface density compared to other methods and it is easier to be done. However, AuNRs dispersions synthesized by wet chemical methods such as SMGM often contain gold nanospheres (AuNSs) as their byproduct [15]. Hence, centrifugation process is required to separate the nanorods with its by-product to form high quality gold nanorods on the film and further will be used as sensing material in LPSR sensor.

\section{Methodology}

\subsection{Chemicals}

Hydrogen tetrachloroaurate (III) hydrate (HAuCl4), hexadecyltrimethylammonium bromide (CTAB) and sodium borohydride (NaBH4), L-ascorbic acid were purchased from Sigma Aldrich (USA) while silver nitrate (AgNO3) were purchased from Honeywell Research Chemicals. The deionized water was used for preparation of the solution.

\subsection{AuNRs synthesis}

The synthesis of gold nanorods by using seed mediated growth method (SMGM) are divided into two parts which are the seeding and the growth process. In the seeding process, the seed solution is prepared by mixing $0.2 \mathrm{M}, 5 \mathrm{~mL}$ CTAB solution with $0.0005 \mathrm{M}, 5$ $\mathrm{mL}$ of $\mathrm{HAuCl} 4$ with icy cold $\mathrm{NaBH} 4$ which ratios are $0.01 \mathrm{M}$, $0.6 \mathrm{~mL}$. The solution is stirred until a brownish pale-yellow coloured solution is produced. After the solution is stirred, it was kept under the temperature of $25^{\circ} \mathrm{C}$ and let to grow for 2 hours. The growth solution is prepared by using $0.001 \mathrm{M}, 15 \mathrm{~mL}$ of $\mathrm{HAuCl} 4$ mixed with $0.004 \mathrm{M}, 0.60 \mathrm{~mL}$ of $\mathrm{AgNO} 3$ and $0.2 \mathrm{M}, 15 \mathrm{~mL}$ of CTAB. After gentle mixing of the solution, $0.0788 \mathrm{M}, 0.21 \mathrm{uL}$ of ascorbic acid is then added. Ascorbic acid acts as a mild reducing agent that changes the yellow coloured solution to colourless. The final and most important step is the addition of $0.216 \mathrm{~mL}$ of the seed solution into the growth solution. The colour changes are observed gradually in the period of 20 to 30 minutes. The sample is then centrifuged for 15 minutes separately according to their respective speed. The samples are labelled as $\mathrm{C} 1, \mathrm{C} 2, \mathrm{C} 3, \mathrm{C} 4, \mathrm{C} 5$ and C6 for $2500 \mathrm{rpm}, 3000 \mathrm{rpm}, 3500 \mathrm{rpm}, 4000 \mathrm{rpm}, 4500 \mathrm{rpm}$ and $5000 \mathrm{rpm}$. The supernatant of the solution is removed after centrifugation and $5 \mathrm{~mL}$ of DI water is added to the sedimentation pellets at the bottom of the tube. The process is then repeated once more and the last pellet of AuNRs is added $0.5 \mathrm{~mL}$ of DI water. The sedimentation liquid is dropped on top of the substrate before it was annealed for 60 minutes at $100^{\circ} \mathrm{C}$.

\section{Result and Discussion}

\subsection{Physical analysis}

The recipe for gold nanorods (AuNRs) synthesis has been obtained and modified from the previous study by Nikoobakht et. al. [4]. Firstly, the physical colour change of AuNRs are observed. The ascorbic acid acts as a mild reducing agent to change the colour of the solution from yellow to transparent. The colour changes gradually to dark purple within 20 to 30 minutes after the seed was added into the growth solution. All the samples were characterized by using UV-Vis before the centrifugation process proceeds. The sample was centrifuged for 15 minutes separately according to their respective speed. The supernatant of the solution was removed after centrifugation and $5 \mathrm{~mL}$ of DI water is added to the sedimentation pellets at the bottom of the tube. The process is then repeated one more time and then $0.5 \mathrm{~mL}$ of DI water were added to the last centrifuged pellet of AuNRs. The sedimentation liquid was dropped on top of the substrate before it was annealed for 60 minutes at $100^{\circ} \mathrm{C}$. Figure 1 shows the change in colour of the
AuNRs after the centrifugation process. The centrifugation speed clearly affects the physical colour of the solution after the centrifugation. Higher centrifugation speed shows a darker hue of purple whereas the lower centrifugation speed produces a lighter hue of purple. This difference is due to the sedimentation of nanoparticles which consists of AuNRs and also its by-product accumulated at the bottom of the tube.
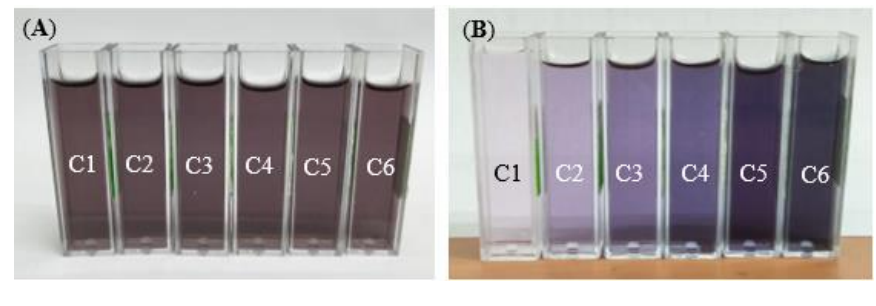

Figure 1: Image of the cuvette shows the result of C1 (2500 rpm), C2 (3000 rpm), C3 (3500 rpm), C4 (4000 rpm), C5 (4500 rpm) and C6 (5000 $\mathrm{rpm}$ ) which is obtained (A) before centrifugation; (B) after centrifugation according to the assigned centrifugation speed.

\subsection{Optical analysis}

The analysis on the growth solution of AuNRs was done to ensure all samples have the same absorption spectra before the speed variation. The by-product of the solutions consists of mostly gold nanospheres (AuNSs) which only have one surface plasmon peak, located at the wavelength of about $520 \mathrm{~nm}$ whereas AuNRs have both transverse plasmon band in the range of $500 \mathrm{~nm}$ to $550 \mathrm{~nm}$ and longitudinal plasmon band from $700 \mathrm{~nm}$ to $800 \mathrm{~nm}$. The intensity of the t-LSPR is only at 0.86 . Meanwhile, the intensity of the 1-LSPR is at 2.37 which are visibly higher than the t-LSPR peak. The resulting absorption spectra of AuNRs are plotted in Figure 2 (A) and it shows two absorption peaks which consists of a weaker transverse plasmon band (t-LSPR) and a stronger longitudinal plasmon band (1-LSPR).

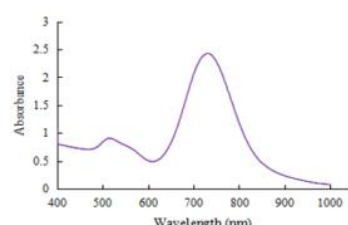

(A)

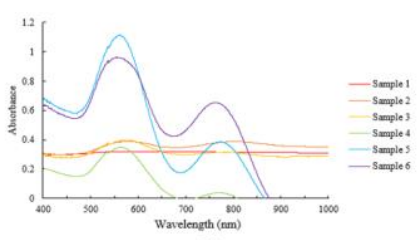

(B)
Figure 2: The absorbance spectra graph of the gold nanorods (A) Before centrifugation and (B) After centrifugation.

Meanwhile for the UV-Vis analysis for the solution after the centrifugation is displayed in Figure 2 (B). The absorbance spectra of each samples after centrifugation is observed and it clearly shows that the intensity of 1-LSPR is increasing when the centrifuge speed is increasing. The sample $\mathrm{C} 1$ produces a straight line for the absorbance spectra graph. This is because the sample $\mathrm{C} 1$ is too diluted which causes the UV-Vis to produce a straight line. Both samples C2 and C3 have curves with smaller t-LSPR and 1-LSPR peaks. Meanwhile, both sample C4 and C5 show higher t-LSPR peak than the 1-LSPR. This result shows that the presence of both AuNRs and its by-product on the sample solution. But the 1-LSPR peak in sample C6 can be observed to be higher than sample C5 and it corresponds to weaker t-LSPR peak. Table 1 shows the analysis of the spectrum peak and wavelength of each of the samples after centrifugation has been done. The peak and the intensity of sample $\mathrm{C} 1$ is not visible because it has no peaks. The wavelength for both the t-LSPR and l-LSPR is red shifted. Ideally, the 1-LSPR should be blue shifted but it is red shifted due to the nonhomogenous properties of the produced AuNRs. 
Table 1: The peak shift of the Localized Surface Plasmon Resonance (LSPR)

\begin{tabular}{|c|c|c|c|c|}
\hline \multirow{2}{*}{ Sample } & \multicolumn{2}{|c|}{ t-LSPR } & \multicolumn{2}{c|}{ l-LSPR } \\
\cline { 2 - 5 } & Intensity & $\begin{array}{c}\text { Wavelength } \\
\text { (nm) }\end{array}$ & Intensity & $\begin{array}{c}\text { Wavelength } \\
\text { (nm) }\end{array}$ \\
\hline C1 & - & - & - & - \\
\hline C2 & 0.387 & 577 & 0.386 & 799 \\
\hline C3 & 0.398 & 573 & 0.315 & 785 \\
\hline C4 & 0.349 & 564 & 0.037 & 774 \\
\hline C5 & 1.116 & 560 & 0.385 & 774 \\
\hline C6 & 0.958 & 560 & 0.653 & 766 \\
\hline
\end{tabular}

\subsection{Morphology analysis}

The surface density of AuNRs were measured to calculate the number of particles per unit area of the surface. It is used mainly to observe the effect of the centrifugation speed towards the density of AuNRs on the substrate surface. The mean percentage was calculated based on the images obtained from FE-SEM. Table 3 shows the surface density percentage of the samples. Based on the analysis, the surface density of the AuNRs that has been annealed on top of the substrate is directly proportional to the rotational speed. The percentage of AuNRs density of sample $\mathrm{C} 1$ centrifuged at 2,500 rpm is noticeably smaller than the sample C6 centrifuged at 5,000 rpm. The sample C1 has only $7.45 \%$ of AuNRs whereas sample C6 shows much higher AuNRs surface density percentage at $15.56 \%$. This analysis shows that the higher centrifugation speed will have a higher percentage of extracted AuNRs deposited at the surface of the substrate.

Table 2: Average aspect ratio of AuNRs and percentage of surface density per samples

\begin{tabular}{|c|c|c|}
\hline Sample & Aspect Ratio & $\begin{array}{c}\text { Surface Density of AuNRs } \\
(\mathbf{\%})\end{array}$ \\
\hline C1 & $2.37 \pm 0.04$ & 7.45 \\
\hline C2 & $2.38 \pm 0.06$ & 7.86 \\
\hline C3 & $2.42 \pm 0.05$ & 10.89 \\
\hline C4 & $2.43 \pm 0.04$ & 12.68 \\
\hline C5 & $2.72 \pm 0.06$ & 13.76 \\
\hline C6 & $2.89 \pm 0.03$ & 15.56 \\
\hline
\end{tabular}

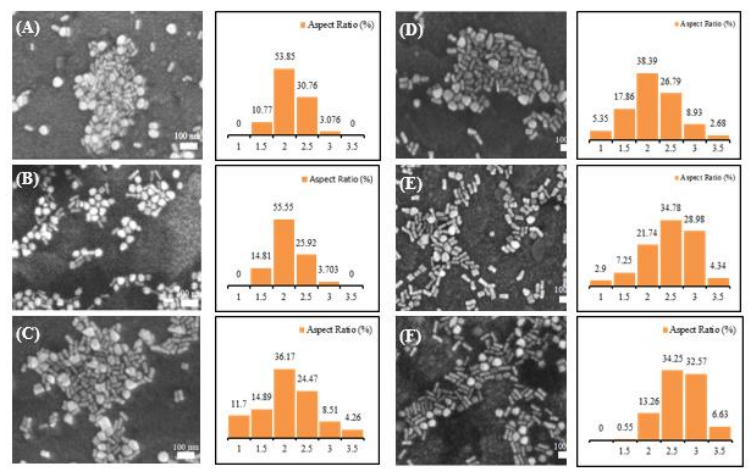

Figure 3: FE-SEM images of AuNRs which the centrifugation speed is varied and the bar chart shows the percentage of aspect ratio shown in the graph; (A) sample C1; (B) sample C2; (C) sample C3; (D) sample C4; (E) sample C5; (F) sample C6

The FE-SEM result in Figure 3 shows a distinct difference in the separation of the AuNRs. Sample C1 with low centrifugation speed results in a congregation of both AuNRs and its by-product in one place. As the rotational speed increases, it is observed that the AuNRs becomes more separated from each other and the amount of AuNRs is increasing. The diameter of the AuNRs are also increasing as well. The aspect ratio with a range in between 3.00 to 3.49 shows the obvious change from only $3.08 \%$ at the speed of 2,500 rpm and it rises to $32.57 \%$ at the speed of 5,000 rpm. Meanwhile, the AuNRs with the aspect ratio with the range of 3.50 to 4.00 also can be seen as increasing to $6.63 \%$ at sample
C6 with a comparison to the $0 \%$ which was observed from sample C1. The lower aspect ratio of AuNRs also can be observed decreasing with the increasing rotations per minute. Sample C1 shows a higher percentage of AuNRs with the aspect ratio of 1.50 which is at $10.77 \%$ while the percentage decreases at sample C6 which is only at $0.55 \%$. This can be proved by calculating the average aspect ratio for each sample that was centrifuged.

The average aspect ratio of each sample is also increasing as the rotational speed of the centrifuge is increased. This is because the high centrifugation speed allows heavier and longer AuNRs to be extracted at the bottom of the tube [15]. As shown in Figure 3, the percentage of higher aspect ratio increases with increasing centrifugation speed. The impact of applying 5,000 rpm is noticeable as the morphology of the AuNRs is becoming more uniform than the lower centrifugation speed. The graph distribution is noticed shifting more to the right side where a higher aspect ratio of AuNRs lies. The dominant aspect ratio during low centrifugation is in the range of 1.50 to 2.00 . As the speed of the centrifuge increases, the dominant aspect ratio of AuNRs changes to a bigger aspect ratio which is in the range of 2.50 to 3.00 . The result shows that the aspect ratio of the samples is increased when the centrifugation speed is raised and at higher centrifugation speed, the higher aspect ratio of AuNRs are separated from their by-product.

\section{Conclusion}

Samples exposed to higher centrifugation speed shows darker purple hue solution which contains more AuNRs compared to samples exposed to lower centrifugation speed with the same centrifugation time. The AuNRs shows two localized surface plasmon resonance (LSPR) peaks which composed of transverse peak (tLSPR) and longitudinal peak (1-LSPR). The analysis on absorbance spectra graph shows the blue shift in both the t-LSPR and 1LSPR. The 1-LPSR peak is ideally red shifted due to the nonhomogenous properties of the extracted AuNRs. The surface density and the aspect ratio of the AuNRs are highly affected by the centrifugation speed. The surface density increases from $7.45 \%$ to $15.56 \%$ with the increasing centrifugation speed. Meanwhile, the aspect ratio of samples at centrifugation speed of 2,500 rpm is $2.37 \pm 0.04$ and it increases to $2.89 \pm 0.03$ at centrifugation speed of $5,000 \mathrm{rpm}$. The analysis shows that more AuNRs with higher aspect ratio are separated by increasing the centrifugation speed.

\section{Acknowledgement}

The authors acknowledge invaluable contribution from Microelectronics \& Nanotechnology-Shamsuddin Research Centre (MiNTSRC), Universiti Tun Hussein Onn Malaysia. The authors would to thank Associate Professor Dr. Mohd Zainizan Bin Sahdan for facilities used in this experiment. This work was supported by the Universiti Tun Hussein Onn Malaysia under Contract Research Grant (U565-UTHM) and GPPS (U989-UTHM).

\section{References}

[1] Morsin M, Salleh MMM, Umar MAA \& Sahdan MZ, "Gold Nanoplates for a Localized Surface Plasmon Resonance-Based Boric Acid Sensor", Sensors, Vol.17, Vol.5, (2017), pp. 947

[2] Gulati A, Liao H \& JH Hafner, "Monitoring Gold Nanorod Synthesis by Localized Surface Plasmon Resonance", J. Phys. Chem. B, Vol. 110, No.45, (2006), pp. 22323-22327.

[3] Wang J, Zhang HZ, Li RS \& Huang CZ, "Localized surface plasmon resonance of gold nanorods and assemblies in the view of biomedical analysis", Anal. Chem., Vol.80, (2016), pp. 429-443.

[4] Nikoobakht B \& El Sayed, "Preparation and Growth Mechanism of Gold Nanorods (NRs) Using Seed - Mediated Growth Method", Chem. Mater., Vol.15, No.16, (2003), pp. 1957-1962.

[5] AJ Haes, Stuart DA, Nie S \& Duyne RPV, "Using solution-phase nanoparticles, surface-confined nanoparticle arrays and single na- 
noparticles as biological sensing platforms", J. Fluoresc., Vol.14, No 4, (2004), pp. 355-367.

[6] Morsin M, Umar AA, Salleh MM, Majlis BY, "High sensitivity localized surface plasmon resonance sensor of gold nanoparticles: Surface density effect for detection of boric acid. In Semiconductor Electronics (ICSE), 2012 10th IEEE International Conference on (2012)pp. 352-356,

[7] Nengsih S, Umar AA, Salleh MM \& Oyama M, "Detection of for maldehyde in water: A shape-effect on the plasmonic sensing properties of the gold nanoparticles", Sensors, Vol.12, No.8, (2012), pp. 10309-10325.

[8] Ma ZY, Xia HX, Liu YP, Liu B, Chen W \& Di Zhao Y, “Applications of gold nanorods in biomedical imaging and related fields", Chinese Sci. Bull., Vol.58, No.21, (2013), pp. 2530-2536.

[9] Song JH, Kim F, Kim D \& Yang P "Crystal overgrowth on gold nanorods: Tuning the shape, facet, aspect ratio, and composition of the nanorods", Chem. - A Eur. J., Vol.11, No.3, (2005), pp. 910 916.

[10] Boksebeld M, Blanchard NP, Jaffal A, Chevolot Y \& Monnier V, "Shape-selective purification of gold nanorods with low aspect ratio using a simple centrifugation method", Gold Bull., Vol.50, No.1, (2017), pp. 69-76.

[11] Kimling J, Maier M, Okenve B, Kotaidis V, Ballot H \& Plech A, "Turkevich Method for Gold Nanoparticle Synthesis Revisited", J. Phys. Chem. B, Vol.110, (2006), pp. 15700-15707,

[12] Uehara A, Booth SG, Chang SY, Schroeder SL, Imai T, Hashimoto T, Mosselmans JF, Dryfe RA, "Electrochemical Insight into the Brust-Schiffrin Synthesis of Au Nanoparticles", J. Am. Chem. Soc., Vol.137, No.48, (2015), pp. 15135-15144.

[13] Rama S, Perala K \& Kumar S, "On the Mechanism of Metal Nanoparticle Synthesis in the Brust- Schiffrin Method", Langmuir, Vol.29, No.31, (2013), pp. 9863-9873.

[14] Ma X, Wang MC, You F, Feng J \& Zhao X, "Optical properties and microstructure of $\mathrm{Au}$ nanorods via seed solution amount variation synthesized by seed-mediated growth method", J. Alloys Compd., Vol.649, (2015), pp. 617-624.

[15] Sharma V, Park K \& Srinivasarao M, "Shape separation of gold nanorods using centrifugation", Vol.106, No.13, (2009), pp. 49814985 . 